\title{
Relationship between Presence of Visceral Infarction and Functional Outcome among Patients with Acute Ischemic Stroke
}

\author{
Pirouz Piran a,b Yahya B. Atalay ${ }^{a, b}$ Ajay Gupta ${ }^{a, c}$ Praneil Patel ${ }^{a, c}$ Santosh \\ B. Murthy ${ }^{a, b}$ Babak B. Navia,b Hooman Kamel ${ }^{a, b} \quad$ Alexander E. Merkler ${ }^{a, b}$ \\ ${ }^{a}$ Clinical and Translational Neuroscience Unit, Feil Family Brain and Mind Research Institute, New York, NY, USA; \\ ${ }^{b}$ Department of Neurology, Weill Cornell Medicine, New York, NY, USA; 'Department of Radiology, Weill Cornell \\ Medicine, New York, NY, USA
}

\section{Keywords}

Visceral infarction · Stroke · Functional outcomes ·

Cardioembolism $\cdot$ Renal infarction $\cdot$ Splenic infarction

\begin{abstract}
Introduction: Evidence of visceral infarction is often found in patients with acute ischemic stroke. It remains uncertain whether there exists a relationship between visceral infarction and functional outcomes among patients with stroke. Objective: The aim of this study was to evaluate whether evidence of visceral infarction is associated with functional outcomes among patients with stroke. Methods: Among patients with acute ischemic stroke enrolled in the Cornell AcutE Stroke Academic Registry (CAESAR) from 2011 through 2016, we included those with a contrast-enhanced abdominal computed tomographic scan within 1 year of admission. Our outcome was ambulatory status at discharge from acute stroke hospitalization, categorized as walking without assistance, walking with assistance, and unable to walk. We used ordinal logistic regression to examine the association between visceral infarction and discharge ambulatory status after adjustment for demographics, stroke risk factors, stroke severity (NIH Stroke Scale), and stroke subtype. Results:
\end{abstract}

Among 2,116 ischemic stroke patients registered in CAESAR from 2011 to 2016, 259 had contrast-enhanced abdominal computed tomographic imaging, of whom 48 (19\%) had evidence of visceral infarction. After adjustment for demographics, stroke risk factors, stroke severity, and stroke subtype, the presence of visceral infarction was associated with a worse ambulatory status at discharge (global OR for better ambulatory status, $0.4 ; 95 \% \mathrm{Cl}, 0.2-1.0, p=0.046)$. Conclusions: We found that the presence of visceral infarction was associated with poor functional outcomes at the time of hospital discharge. These findings suggest that such findings are not necessarily benign and are at the least a marker of poor outcomes.

(C) 2020 The Author(s).

Published by S. Karger AG, Basel

\section{Introduction}

Visceral infarctions are often incidentally detected in patients with acute ischemic stroke or TIA [1]. Among an autopsy study of patients with fatal ischemic stroke, onefifth had evidence of visceral infarctions; renal and splenic infarctions were the most frequent types of visceral infarctions [2]. Visceral infarctions may be a marker of a 
Fig. 1. Study flow diagram. Study flow diagram detailing patient selection. CAESAR, Cornell AcutE Stroke Academic Registry; CT, computed tomography.

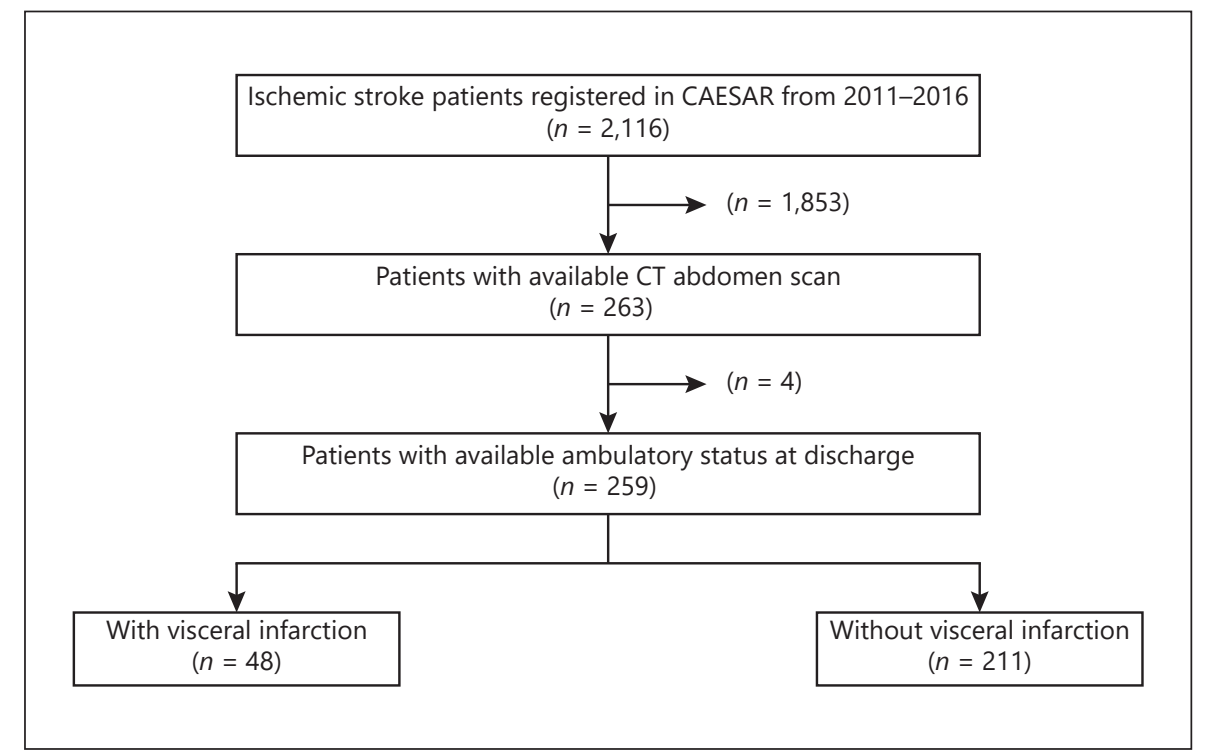

central embolic source as they are more often detected in patients with cardioembolic stroke or embolic stroke of undetermined source [3]. Despite their frequency, the clinical significance of visceral infarctions remains poorly understood. We hypothesized that visceral infarctions would be associated with poor short-term functional outcomes and, therefore, examined the association between visceral infarctions and functional outcomes after ischemic stroke.

\section{Patients and Methods}

\section{Design and Population}

We performed a cross-sectional study of acute ischemic stroke patients who were prospectively registered into the Cornell AcutE Stroke Academic Registry (CAESAR) from January 2011 through December 2016. All patients hospitalized for acute ischemic stroke at the NewYork-Presbyterian Hospital/Weill Cornell Medical Center are prospectively enrolled in the American Heart Association's Get With The Guidelines - Stroke Registry. Trained hospital analysts prospectively collect data on demographics, vascular risk factors and comorbidities, stroke severity (NIHSS), and in-hospital treatments and outcomes. CAESAR combines the Get With The Guidelines data with additional retrospectively collected clinical, laboratory, and radiographic data. For this analysis, we included all stroke patients who had a contrast-enhanced abdominal computed tomographic scan within 1 year of stroke hospitalization. The data that support the findings of this study are available from the corresponding author upon reasonable request. Our institutional review board approved this study.

Visceral Infarction and Outcomes in Stroke

\section{Measurements}

Patients' underlying stroke mechanism was determined by a panel of board-certified neurologists who adjudicated the cause of stroke per the Trial of ORG 10172 in Acute Stroke Treatment classification [4] and per the proposed criteria for embolic stroke of undetermined source [5]. One board-certified radiologist blinded to patients' ambulatory status outcomes reviewed contrast-enhanced abdominal computed tomography (CT) imaging. Visceral infarction was defined as the presence of infarction in the spleen, kidneys, or both within 1 year of stroke admission. Infarction was identified as the presence of peripheral wedge-shaped hypoattenuation on abdominal CT scan. Cortical rim enhancement/sign was used to differentiate ischemic kidney infarction from pyelonephritis. Ultrasound results were used to assist with the diagnosis of infarction when available. If multiple abdominal CT scans were eligible for evaluation, the study closest in time to the stroke hospitalization was evaluated. As splenic pathology is difficult to characterize definitively in the arterial phase, only CT scans with a portal venous phase were utilized for evaluation. We used discharge ambulatory status as a surrogate for functional outcomes. Ambulatory status was recorded prospectively in all patients at the time of discharge and was categorized as (1) walking without assistance, (2) walking with assistance, or (3) unable to walk. Patients who died or who were discharged to hospice were considered unable to walk.

\section{Statistical Analysis}

The $\chi^{2}$ or Fisher's exact test were used to compare categorical variables and the $t$ test was used to compare continuous variables. Ordinal logistic regression was used to assess whether the presence of visceral infarction was associated with good functional outcome at the time of discharge after adjustment for demographics, stroke risk factors, stroke severity (NIHSS), and stroke subtype. In a sensitivity analysis, we limited our cohort to patients who underwent abdominal CT within 14 days of stroke hospitalization. All statistical analyses were performed by A.E.M. using Stata/MP (version 14, College Station, TX, USA). The alpha threshold for statistical significance was 0.05 . 


\section{Results}

Among 2,116 ischemic stroke patients registered in CAESAR from 2011 to 2016, 263 patients had a contrastenhanced abdominal CT scan within 1 year of stroke hospitalization. Of these 263 patients, 259 had available information regarding ambulatory status at discharge and thus were included in our final analysis (Fig. 1). Patients' mean age was $69.3( \pm 15.6)$ years and $51 \%$ were women. The most common indications for obtaining abdominal CT imaging was evaluation for GI disorder $(n=78)$, malignancy $(n=77)$, and vascular disease $(n=33)$. There were 48 patients (19\%) who had evidence of visceral infarction. Baseline characteristics were similar between the patients with visceral infarction and those without (Table 1). There was no difference in length of stay in patients with and without visceral infarction (11.6 vs. 11.6 days, $p=1.0$ ).

Among the 211 patients without visceral infarction, at discharge, $126(60 \%)$ could walk without assistance, 34 (16\%) could walk with assistance, and 51 (24\%) could not walk. In comparison, among the 48 patients with visceral infarction, at discharge, 18 (36\%) could walk without assistance, 9 (19\%) could walk with assistance, and 21 (44\%) could not walk (Fig. 2). After adjustment for demographics, stroke risk factors, stroke severity, and stroke subtype, the presence of visceral infarction was associated with a worse ambulatory status at discharge (global OR for better ambulatory status, $0.4 ; 95 \% \mathrm{CI}, 0.2-1.0 ; p=0.046$ ).

We performed 1 sensitivity analysis in which we limited our analysis to patients who underwent an abdominal CT scan within 14 days of their admission for stroke $(n=116)$. Among these 116 patients, 26 (22\%) had evidence of visceral infarction. Among the 90 patients without visceral infarction, at discharge, $50(56 \%)$ could walk without assistance, 11 (12\%) could walk with assistance, and 29 (32\%) could not walk. In comparison, among the 26 patients with visceral infarction, at discharge, $6(23 \%)$ could walk without assistance, 6 (23\%) could walk with assistance, and 14 (54\%) could not walk. After adjustment for demographics, stroke risk factors, stroke severity, and stroke subtype, the presence of visceral infarction was associated with a worse ambulatory status at discharge (global OR for better ambulatory status, $0.2 ; 95 \% \mathrm{CI}, 0.05-1.00 ; p=0.05)$.

\section{Discussion/Conclusion}

Among patients in a prospective stroke registry, we found that the presence of visceral infarction was associated with worse functional outcomes at the time of hos-
Table 1. Baseline characteristics of patients with acute ischemic stroke who had contrast-enhanced abdominal CT imaging, stratified by the presence of visceral infarction

\begin{tabular}{|c|c|c|c|}
\hline Characteristic* & $\begin{array}{l}\text { Visceral } \\
\text { infarction } \\
(n=48)\end{array}$ & $\begin{array}{l}\text { No visceral } \\
\text { infarction } \\
(n=211)\end{array}$ & $\begin{array}{l}p \\
\text { value }\end{array}$ \\
\hline Age, mean (SD), years & $69.3(14.8)$ & $69.3(15.6)$ & 0.4 \\
\hline Female & $23(47.9)$ & $110(52.1)$ & 0.7 \\
\hline \multicolumn{4}{|l|}{ Race } \\
\hline White & $43(89.6)$ & $179(84.8)$ & \multirow{4}{*}{0.03} \\
\hline Black & $2(4.2)$ & $20(9.5)$ & \\
\hline Hispanic & $2(4.2)$ & $0(0)$ & \\
\hline Other & $1(2.1)$ & $12(5.7)$ & \\
\hline Active tobacco use & $5(10.4)$ & $18(8.5)$ & 0.01 \\
\hline NIHSS $^{\dagger}$ (median, IQR) & $7(3-19)$ & $5(2-11)$ & 0.4 \\
\hline \multicolumn{4}{|l|}{ Stroke subtype } \\
\hline $\begin{array}{l}\text { Large artery } \\
\text { atherosclerosis }\end{array}$ & $2(4.2)$ & $27(12.8)$ & \multirow{5}{*}{0.2} \\
\hline Cardioembolic & $24(50.0)$ & $57(27.0)$ & \\
\hline Small vessel occlusion & $0(0)$ & $17(8.1)$ & \\
\hline Other & $3(6.3)$ & $10(4.7)$ & \\
\hline Cryptogenic & $19(39.6)$ & $100(47.4)$ & \\
\hline Diabetes mellitus & $12(25.0)$ & $55(25.6)$ & 0.4 \\
\hline Dyslipidemia & $23(47.9)$ & $90(42.7)$ & 0.1 \\
\hline Hypertension & $32(66.7)$ & $137(64.9)$ & 0.1 \\
\hline Coronary artery disease & $12(25.0)$ & $45(21.3)$ & 0.4 \\
\hline Congestive heart failure & $3(6.3)$ & $9(4.3)$ & 0.6 \\
\hline CKD & $0(0.0)$ & $6(2.8)$ & 1.0 \\
\hline Atrial fibrillation & $10(20.8)$ & $37(17.5)$ & 0.8 \\
\hline Peripheral vascular disease & $4(8.3)$ & $14(6.36)$ & 1.0 \\
\hline Valvular disease & $1(2.1)$ & $7(3.3)$ & 1.0 \\
\hline Prior stroke & $16(33.3)$ & $45(21.3)$ & 0.1 \\
\hline
\end{tabular}

CT, computed tomography; SD, standard deviation; IQR, interquartile range; $\mathrm{CKD}$, chronic kidney disease. ${ }^{*}$ Data are presented as number (\%) unless otherwise specified. ${ }^{\dagger}$ National Institutes of Health Stroke Scale.

pital discharge. Our results suggest that visceral infarction may be a negative prognostic marker.

Visceral infarctions are often incidentally detected in patients with acute ischemic stroke. Recently, we have reported that visceral infarctions are more often detected in patients with cardioembolic or cryptogenic stroke subtypes [3]. Little is known about the mechanisms and significance of these commonly detected lesions. In this context, our findings provide novel data suggesting that visceral infarctions are not a benign, incidental finding and may serve as a marker of disease severity. It may be that visceral infarction is associated with poor functional outcomes because they indicate an active, central embolic source. Future studies should examine whether ischemic stroke patients with visceral infarction might preferen- 
Fig. 2. Functional outcomes at discharge among patients with and without visceral infarction. Distribution of functional outcomes in patients with acute ischemic stroke according to the presence of visceral infarction.

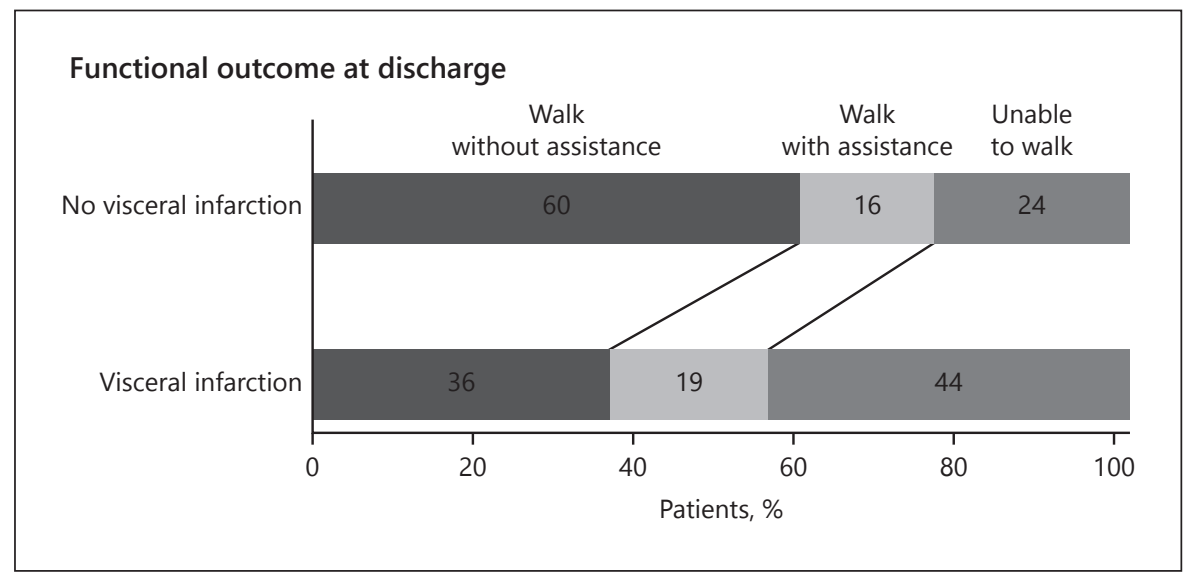

tially benefit from a more aggressive antithrombotic strategy to prevent recurrent embolization.

Our study has several important limitations. First, this was a retrospective cohort of acute ischemic stroke patients at a single tertiary care center and, therefore, the results may not generalize to all patients with stroke. Second, only stroke patients who underwent abdominal CT scans were included in this analysis and, thus, our findings may be susceptible to selection bias. Third, our outcome was ambulatory status at hospital discharge and, thus, the relationship between visceral infarction and long-term outcomes is uncertain, though data suggest that hospital discharge outcomes are reasonable surrogates for long-term functional outcomes among patients with acute ischemic stroke [6-10]. Fourth, we included patients with visceral infarction within 1 year of acute stroke admission and there is a possibility that the visceral infarctions were not present at the time of the stroke. However, the main study result was materially unchanged in a sensitivity analysis that restricted the cohort to patients who underwent abdominal CT scan within 14 days of stroke hospitalization.

In conclusion, the presence of visceral infarction appears to be associated with poor outcomes among patients with acute ischemic stroke. Our findings suggest that visceral infarction should not be dismissed as an incidental finding among patients with acute ischemic stroke.

\section{Acknowledgement}

The authors are grateful to Kelsey Lansdale for her editing and clerical assistance.

Visceral Infarction and Outcomes in Stroke

\section{Statement of Ethics}

Ethical approval for this study was obtained from Weill Cornell Medicine institutional review board.

\section{Disclosure Statement}

Dr. Navi serves as a DSMB member for the PCORI-funded TRAVERSE trial and has received personal fees for medicolegal consulting on stroke. Dr. Kamel serves as co-PI for the NIH-funded ARCADIA trial which receives in-kind study drug from the BMS-Pfizer Alliance and in-kind study assays from Roche Diagnostics, serves as Deputy Editor for JAMA Neurology, serves as a steering committee member of Medtronic's Stroke AF trial (uncompensated), serves on an end point adjudication committee for a trial of empagliflozin for Boehringer-Ingelheim, and has served on an advisory board for Roivant Sciences related to Factor XI inhibition. Dr. Merkler has received personal fees for medicolegal consulting on stroke.

\section{Funding Sources}

Dr. Murthy is supported by the NIH/NINDS grant K23NS105948 and the Leon Levy Foundation. Dr. Navi is supported by NIH grants K23NS091395 and R01HL144541, NIDILRR grant 90REGE0012-01-11, and the Florence Gould Endowment for Discovery in Stroke. Dr. Merkler is supported by AHA grant 18CDA34110419 and the Leon Levy Foundation.

\section{Author Contributions}

PPiran and AED researched literature and conceived the study. AEM was involved in obtaining ethical approval and performed the statistical analyses. PPiran and PPatel collected data. PPiran wrote the first draft of the manuscript. All authors reviewed and edited the manuscript and approved the final version of the manuscript. 


\section{References}

1 Slaoui T, Klein IF, Guidoux C, Cabrejo L, Meseguer E, Abboud H, et al. Prevalence of subdiaphragmatic visceral infarction in cardioembolic stroke. Neurology. 2010;74(13):1030-2.

2 Abboud H, Labreuche J, Gongora-Riverra F, Jaramillo A, Duyckaerts C, Steg PG, et al. Prevalence andand determinants of subdiaphragmatic visceral infarction in patients with fatal stroke. Stroke. 2007;38(5):1442-6.

3 Finn C, Hung P, Patel P, Gupta A, Kamel H. Relationship between visceral infarction and ischemic stroke subtype. Stroke. 2018;49(3): 727-9.

4 Adams Jr HP, Bendixen BH, Kappelle LJ, Biller J, Love BB, Gordon DL, et al. Classification of subtype of acute ischemic stroke. Definitions for use in a multicenter clinical trial. TOAST. Trial of Org 10172 in Acute Stroke Treatment. Stroke. 1993;24(1):35-41.
5 Hart RG, Diener HC, Coutts SB, Easton JD, Granger CB, O’Donnell MJ. Cryptogenic strokes of undetermined source: the case for a new clinical construct. Lancet Neurol. 2014; 13(4):429-38.

6 Brown AW, Therneau TM, Schultz BA, Niewczyk PM, Granger CV, et al. Measure of functional independence dominates discharge outcome prediction after inpatient rehabilitation for stroke. Stroke. 2015;46(4): 1038-44.

7 Granger HS, Nakayama H, Raaschou HO, Vive-Larsen J, Støier M, Olsen TS. Outcome and time course of recovery in stroke. Part I: outcome. The copenhagen stroke study. Arch Phys Med Rehabil. 1995;76:399-405.
8 Veerbeek JM, Van Wegen EE, HarmelingVan der Wel BC, Kwakkel G. Is accurate prediction of gait in nonambulatory stroke patients possible within 72 hours poststroke? The EPOS study. Neurorehabil Neural Repair. 2011;25(3):268-74.

9 Jørgensen HS, Nakayama $\mathrm{H}$, Raaschou $\mathrm{HO}$, Olsen TS. Recovery of walking function in stroke patients: the Copenhagen Stroke Study. Arch Phys Med Rehabil. 1995;76(1): 27-32.

10 Sanchez-Blanco I, Ochoa-Sangrador C, Lopez-Munain L, Izquierdo-Sánchez M, Fermoso-García J. Predictive model of functional independence in stroke patients admitted to a rehabilitation programme. Clin Rehabil. 1999;13(6):464-75. 